Proceedings of International Mathematical Sciences

ISSN:2717-6355, URL:HTTPS://DERGIPARK.ORG.TR/TR/PUB/PIMS

Volume II Issue 2 (2020), Pages 129-135. Doi: 10.47086/Pims.778024

\title{
A NOTE ON THE STABILITY OF SOLUTION FOR ELLIPTIC-SCHRÖDINGER TYPE NONLOCAL BOUNDARY VALUE PROBLEM
}

\author{
YILDIRIM OZDEMIR* AND MECRA ESER** \\ *DUZCE UNIVERSITY, KONURALP CAMPUSS, FACULTY OF ARTS AND SCIENCES, \\ DUZCE, TURKEY. ORCID NUMBER:0000-0003-2767-522X \\ **DUZCE UNIVERSITY, DUZCE, TURKEY. ORCID NUMBER:0000-0002-2779-7190
}

\begin{abstract}
In the present article, a problem for an elliptic-Schrödinger equation with nonlocal boundary value condition is considered. The stability estimates are established for the solution of elliptic-Schrödinger problem. A theorem for stability of the solution of this problem and a conclusion section is presented.
\end{abstract}

\section{INTRODUCTION}

In the present paper, the nonlocal boundary-value problem (NBVP)

$$
\left\{\begin{array}{l}
-\frac{d^{2} u(t)}{d t^{2}}+A u(t)=g(t)(0 \leq t \leq 1), \\
i \frac{d u(t)}{d t}-A u(t)=f(t)(-1 \leq t \leq 0), \\
u(1)=u(-1)+\varphi
\end{array}\right.
$$

for differential equations of elliptic-Schrödinger type in a Hilbert space $H$ with self-adjoint positive definite operator $A$ is considered.

In the literature it is known that various NBVPs for the elliptic-Schrödinger equations can be reduced to the problem (1.1).

Whenever the following conditions are satisfied an abstract function $u(t)$ is called a solution of the problem (1.1):

i. $u(t)$ is twice continuously differentiable on the interval $(0,1]$ and continuously differentiable on the segment $[-1,1]$. The derivative at the endpoints of the segment are understood as the appropriate unilateral derivatives.

2020 Mathematics Subject Classification. 2010 MSC: 65L10, 34B10, 65M12.

Key words and phrases. partial differential equation; nonlocal boundary value problem; stability.

(C)2020 Proceedings of International Mathematical Sciences.

Submitted on August 07th, 2020. Published on 12.30.2020. Communicated by Sahin Uyaver. 
ii. The element $u(t)$ belongs to $D(A)$ for all $t \in[-1,1]$, and the function $A u(t)$ is continuous on the segment $[-1,1]$.

iii. $u(t)$ satisfies the equations and nonlocal boundary condition (1.1).

There are also different type of works on elliptic and Schrödinger equations (see, for example, [13, 14, 15, 16] and references given therein).

Many scientists have been studied the methods of solutions of NBVPs for partial differential equations (PDEs) and PDEs of mixed type extensively (see, 1, 2, 3, 4, [5, 6, 7, 8, 9, 10, 11, 12] and references given therein).

\section{THE MAIN THEOREM ON STABILITY}

In the present section the stability estimates for elliptic-Schrödinger equations are obtained.

Theorem 2.1. Suppose that $\varphi \in D(A)$ and $f(0), g(0) \in H$. Let $f(t)$ be continuously differentiable and $g(t)$ be twice continuously differentiable functions on $[-1,0]$ and $[0,1]$, respectively. Then, there is a unique solution of the problem (1.1) and the following stability inequalities hold:

$$
\begin{gathered}
\max _{-1 \leq t \leq 1}\|u(t)\|_{H} \leq M\left[\|\varphi\|_{H}+\max _{-1 \leq t \leq 0}\|f(t)\|_{H}+\max _{0 \leq t \leq 1}\left\|A^{-1 / 2} g(t)\right\|_{H}\right] \\
\max _{-1 \leq t \leq 1}\left\|\frac{d u(t)}{d t}\right\|_{H}+\max _{-1 \leq t \leq 1}\left\|A^{1 / 2} u(t)\right\|_{H} \\
\leq M\left[\left\|A^{1 / 2} \varphi\right\|_{H}+\max _{-1 \leq t \leq 0}\left\|A^{1 / 2} f(t)\right\|_{H}+\max _{0 \leq t \leq 1}\|g(t)\|_{H}\right] \\
\max _{-1 \leq t \leq 0}\left\|\frac{d u(t)}{d t}\right\|_{H}+\max _{0 \leq t \leq 1}\left\|\frac{d^{2} u(t)}{d t^{2}}\right\|_{H}+\max _{-1 \leq t \leq 1}\|A u(t)\|_{H} \\
\leq M\left[\|A \varphi\|_{H}+\|g(0)\|_{H}+\|f(0)\|_{H}+\max _{0 \leq t \leq 1}\left\|g^{\prime}(t)\right\|_{H}+\max _{-1 \leq t \leq 0}\left\|f^{\prime}(t)\right\|\right]
\end{gathered}
$$

where $M$ is independent of not only $f(t), t \in[-1,0]$ but also $g(t), t \in[0,1]$ and $\varphi$.

Proof. First of all, we will obtain a formula for the solution of problem (1.1). It is known that there are unique solutions of the initial value problems

$$
-\frac{d^{2} u(t)}{d t^{2}}+A u(t)=g(t),(0 \leq t \leq 1), u(0)=u_{0}, u(1)=u_{1},
$$

and

$$
i \frac{d u(t)}{d t}-A u(t)=f(t),(-1 \leq t \leq 0), u(0)=u_{0}
$$

that is,

$$
u(t)=e^{-t A} u_{0}-i \int_{0}^{t} e^{-i(t-s) A} f(s) d s,-1 \leq t \leq 0
$$

and

$$
\begin{gathered}
u(t)=\left(I-e^{-2 A^{1 / 2}}\right)^{-1}\left[\left(e^{-t A^{1 / 2}}-e^{-(-t+2) A^{1 / 2}}\right) u_{0}\right. \\
\left.+\left(e^{-(1-t) A^{1 / 2}}-e^{-(t+1) A^{1 / 2}}\right) u_{1}\right] \\
+\left(I-e^{-2 A^{1 / 2}}\right)^{-1}\left(e^{-(1-t) A^{1 / 2}}-e^{-(t+1) A^{1 / 2}}\right)
\end{gathered}
$$




$$
\begin{gathered}
\times \int_{0}^{1} A^{-1 / 2} 2^{-1}\left(e^{-(1-s) A^{1 / 2}}-e^{-(s+1) A^{1 / 2}}\right) g(s) d s \\
-\int_{0}^{1} A^{-1 / 2} 2^{-1}\left(e^{-(t+s) A^{1 / 2}}-e^{-|t-s| A^{1 / 2}}\right) g(s) d s, 0 \leq t \leq 1,
\end{gathered}
$$

respectively. Using formulas 2.6, 2.7) and nonlocal boundary condition

$$
u(1)=u(-1)+\varphi
$$

we get

$$
\begin{gathered}
u(t)=\left(I-e^{-2 A^{1 / 2}}\right)^{-1}\left[\left(e^{-t A^{1 / 2}}-e^{-(-t+2) A^{1 / 2}}\right) u_{0}\right. \\
\left.+\left(e^{-(1-t) A^{1 / 2}}-e^{-(t+1) A^{1 / 2}}\right)\left(e^{i A} u_{0}-i \int_{0}^{-1} e^{i(1+s) A} f(s) d s+\varphi\right)\right] \\
+\left(I-e^{-2 A^{1 / 2}}\right)^{-1}\left(e^{-(1-t) A^{1 / 2}}-e^{-(t+1) A^{1 / 2}}\right) \\
\times \frac{1}{2} \int_{0}^{1}\left(e^{-(1-s) A^{1 / 2}}-e^{-(s+1) A^{1 / 2}}\right) A^{-1 / 2} g(s) d s \\
-\frac{1}{2} \int_{0}^{1}\left(e^{-(t+s) A^{1 / 2}}-e^{-|t-s| A^{1 / 2}}\right) A^{-1 / 2} g(s) d s, 0 \leq t \leq 1 .
\end{gathered}
$$

Now, using the following

$$
u^{\prime}\left(0^{+}\right)=\frac{1}{i}[A u(0)+f(0)]
$$

we obtain the operator equation

$$
\begin{gathered}
\left\{\left(I-e^{-2 A^{1 / 2}}\right)+i\left(I+e^{-2 A^{1 / 2}}\right) A^{-1 / 2}-2 i A^{-1 / 2} e^{-\left(A^{1 / 2}-i A\right)}\right\} u_{0} \\
=i\left\{\left[-2 A^{-1 / 2} e^{-A^{1 / 2}}\left(i \int_{0}^{-1} e^{i A(-1+s)} f(s) d s+\varphi\right)\right]\right. \\
\quad+A^{-1} e^{-A^{1 / 2}} \int_{0}^{1}\left(e^{-(1-s) A^{1 / 2}}-e^{-(s+1) A^{1 / 2}}\right) g(s) d s \\
\left.+i\left(I-e^{-2 A^{1 / 2}}\right) A^{-1} f(0)+A^{-1}\left(I-e^{-2 A^{1 / 2}}\right) \int_{0}^{1} e^{-s A^{1 / 2}} g(s) d s\right\} .
\end{gathered}
$$

Here, the operator

$$
\left(I-e^{-2 A^{1 / 2}}\right)+i A^{-1 / 2}\left(I+e^{-2 A^{1 / 2}}\right)-2 i A^{-1 / 2} e^{-\left(A^{1 / 2}-i A\right)}
$$

has an inverse,

$$
T=\left[\left(I-e^{-2 A^{1 / 2}}\right)+i A^{-1 / 2}\left(I+e^{-2 A^{1 / 2}}\right)-2 i A^{-1 / 2} e^{-\left(A^{1 / 2}-i A\right)}\right]^{-1}
$$

and

holds.

$$
\left\|A^{-1 / 2} T\right\|_{H \rightarrow H} \leq M
$$

It is needed to obtain a formula for $u(0)$ for the solution of problem 1.1. To do this we must show that $T$ is a bounded operator. Let $A^{1 / 2}=B$. Since

$$
A^{-1 / 2} T=\left[\left(A^{1 / 2}-A^{1 / 2} e^{-2 A^{1 / 2}}\right)+i\left(I+e^{-2 A^{1 / 2}}\right)-2 i e^{-\left(A^{1 / 2}-i A\right)}\right]^{-1}
$$


we have that

$$
=\left[\left(B-B e^{-2 B}\right)+i\left(I+e^{-2 B}\right)-2 i e^{-\left(B-i B^{2}\right)}\right]^{-1}
$$

$$
\left\|A^{-1 / 2} T\right\|_{H \rightarrow H} \leq \sup _{\delta \leq \mu<\infty} \frac{1}{\mu-\mu e^{-2 \mu}+i\left(1+e^{-2 \mu}\right)-2 i e^{-\mu} e^{-i \mu^{2}}} .
$$

Using Euler formula, we get

$$
\beta(\mu)=\mu-\mu e^{-2 \mu}+2 e^{-\mu} \sin \mu^{2}+i\left(I+e^{-2 \mu}-2 e^{-\mu} \cos \mu^{2}\right) .
$$

Taking absolute value of $\beta(\mu)$, we obtain

$$
|\beta(\mu)|=\sqrt{\begin{array}{l}
\mu^{2}+\mu^{2} e^{-4 \mu}+4 e^{-2 \mu} \sin ^{2} \mu^{2}-2 \mu^{2} e^{-2 \mu}+4 \mu e^{-\mu} \sin \mu^{2}-4 \mu e^{-3 \mu} \sin \mu^{2} \\
+1+e^{-4 \mu}+4 e^{-2 \mu} \cos ^{2} \mu^{2}+2 e^{-2 \mu}-4 e^{-\mu} \cos \mu^{2}-4 e^{-3 \mu} \cos \mu^{2}
\end{array}}
$$

Or

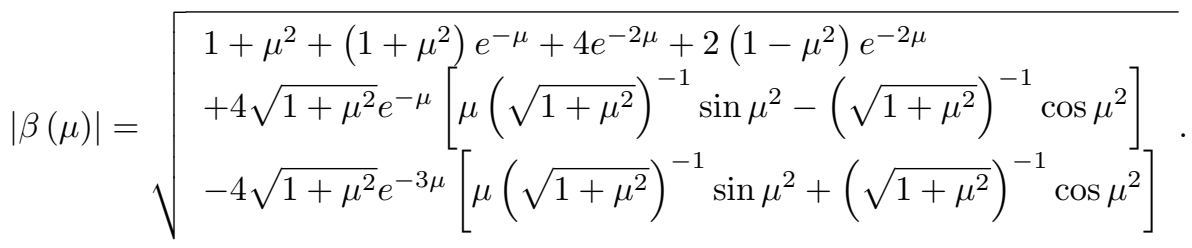

Choosing $\frac{\mu}{\sqrt{1+\mu^{2}}}=\sin \alpha$ and $\frac{1}{\sqrt{1+\mu^{2}}}=\cos \alpha$, we can write

$$
\begin{gathered}
|\beta(\mu)|=\sqrt{\begin{array}{l}
\left(1+\mu^{2}\right)+\left(1+\mu^{2}\right) e^{-4 \mu}+4 e^{-2 \mu}+2\left(1-\mu^{2}\right) e^{-2 \mu} \\
-4 \sqrt{1+\mu^{2}} e^{-\mu} \cos \left(\mu^{2}+\alpha\right)-4 \sqrt{1+\mu^{2}} e^{-3 \mu} \cos \left(\mu^{2}-\alpha\right)
\end{array}} \\
\geq \sqrt{1+\mu^{2}+\left(1+\mu^{2}\right) e^{-4 \mu}+2\left(1-\mu^{2}\right) e^{-2 \mu} 4 \sqrt{1+\mu^{2}} e^{-\mu}-4 \sqrt{1+\mu^{2}} e^{-3 \mu}} .
\end{gathered}
$$

Now, let

$$
\psi(\mu)=\mu^{2}+\left(1+\mu^{2}\right) e^{-4 \mu}+2\left(1-\mu^{2}\right) e^{-2 \mu} 4 \sqrt{1+\mu^{2}} e^{-\mu}-4 \sqrt{1+\mu^{2}} e^{-3 \mu} .
$$

Hence, it is enough to prove the inequality $\psi(\mu)>0$ for $\mu \geq \delta$. It is seen that for sufficiently large $\delta$ the inequality holds. Therefore,

$$
\left\|A^{-1 / 2} T\right\|_{H \rightarrow H} \leq 1 \text {. }
$$

This means that $A^{-1 / 2} T$ is bounded. So,

$$
\begin{gathered}
u(0)=A^{-1 / 2} T\left(I-e^{-2 A^{1 / 2}}\right)\left\{i \int_{0}^{1} A^{-1 / 2} e^{-s A^{1 / 2}} g(s) d s-A^{-1 / 2} f(0)\right\} \\
+T e^{-A^{1 / 2}} A^{-1 / 2}\left\{2 \int_{0}^{-1} e^{i A(-1+s)} f(s) d s-2 i \varphi+i \int_{0}^{1}\right. \\
\left.\times\left(e^{-(1-s) A^{1 / 2}}-e^{-(s+1) A^{1 / 2}}\right) A^{-1 / 2} g(s) d s\right\} .
\end{gathered}
$$

Finally, we have formulas $(2.6),(2.8)$ and $(2.10)$ for the solution of the nonlocal boundary value problem (1.1).

Now, proofs of estimates 2.1, 2.2 and 2.3 will be given. Firstly, we consider 2.1. Using formula 2.10, we get

$$
\|u(0)\|_{H} \leq\left\|A^{-1 / 2} T\right\|_{H \rightarrow H}\left\|I-e^{-2 A^{1 / 2}}\right\|_{H \rightarrow H}
$$




$$
\begin{gathered}
\quad \times\left\{|i| \int_{0}^{1}\left\|e^{-s A^{1 / 2}}\right\|_{H \rightarrow H}\left\|A^{-1 / 2} g(s)\right\|_{H} d s+\left\|A^{-1 / 2}\right\|_{H \rightarrow H}\|f(0)\|_{H}\right\} \\
+\left\|e^{-A^{1 / 2}}\right\|_{H \rightarrow H}\left\|A^{-1 / 2} T\right\|_{H \rightarrow H}\left\{2 \int_{0}^{-1}\left\|e^{i A(-1+s)}\right\|_{H \rightarrow H}\|f(s)\|_{H} d s+2|i|\|\varphi\|_{H}\right. \\
\left.+|i| \int_{0}^{1}\left(\left\|e^{-(1-s) A^{1 / 2}}\right\|_{H \rightarrow H}+\left\|e^{-(s+1) A^{1 / 2}}\right\|_{H \rightarrow H}\right)\left\|A^{-1 / 2} g(s)\right\|_{H} d s\right\}
\end{gathered}
$$

or

$$
\|u(0)\|_{H} \leq M\left[\|\varphi\|_{H}+\max _{-1 \leq t \leq 0}\|f(t)\|_{H}+\max _{0 \leq t \leq 1}\left\|A^{-1 / 2} g(t)\right\|_{H}\right] .
$$

Then, using formulas (2.6) and (2.8), we obtain for $-1 \leq t \leq 0$

$$
\|u(t)\|_{H} \leq M\left[\|\varphi\|_{H}+\max _{-1 \leq t \leq 0}\|f(t)\|_{H}+\max _{0 \leq t \leq 1}\left\|A^{-1 / 2} g(t)\right\|_{H}\right]
$$

and for $0 \leq t \leq 1$

$$
\|u(t)\|_{H} \leq M\left[\|u(0)\|_{H}+\max _{-1 \leq t \leq 0}\|f(t)\|_{H}+\|\varphi\|_{H}+\max _{0 \leq t \leq 1}\left\|A^{-1 / 2} g(t)\right\|_{H}\right] .
$$

Therefore, using estimates 2.12, 2.13) and 2.14, we complete proof of inequality (2.1.).

Secondly, the proof of the estimate $\sqrt{2.2}$ will be obtained. Applying $A^{1 / 2}$ to 2.10 and taking the norm of it, we can write

$$
\left\|A^{1 / 2} u(0)\right\|_{H} \leq M\left[\left\|A^{1 / 2} \varphi\right\|_{H}+\max _{-1 \leq t \leq 0}\left\|A^{1 / 2} f(t)\right\|_{H}+\max _{0 \leq t \leq 1}\|g(t)\|_{H}\right] .
$$

After that, applying $A^{1 / 2}$ to 2.6 and 2.8 and taking norm of them, we obtain

$$
\left\|A^{1 / 2} u(t)\right\|_{H} \leq\left\|A^{1 / 2} u(0)\right\|_{H}+\max _{-1 \leq t \leq 0}\left\|A^{1 / 2} f(t)\right\|_{H},-1 \leq t \leq 0
$$

and

$$
\begin{gathered}
\left\|A^{1 / 2} u(t)\right\|_{H} \leq M\left[\left\|A^{1 / 2} u(0)\right\|_{H}+\max _{-1 \leq t \leq 0}\left\|A^{1 / 2} f(t)\right\|_{H}\right. \\
\left.+\left\|A^{1 / 2} \varphi\right\|_{H}+\max _{0 \leq t \leq 1}\|g(t)\|_{H}\right], 0 \leq t \leq 1 .
\end{gathered}
$$

Combining estimates 2.15), 2.16) and (2.17), we obtain 2.2.

Thirdly, the proof of the estimate 2.3 will be obtained. Using formula 2.6 and integration by parts, we get for $-1 \leq t \leq 0$

$$
u(t)=e^{-t A} u_{0}-A^{-1}\left\{\left[f(t)-e^{-i t A} f(0)\right]-\int_{0}^{t} e^{-i(t-s)} f^{\prime}(s) d s\right\} .
$$

Using formula 2.8 and integration by parts, we get for $0 \leq t \leq 1$

$$
\begin{gathered}
u(t)=\left(I-e^{-2 A^{1 / 2}}\right)^{-1}\left[\left(e^{-t A^{1 / 2}}-e^{-(-t+2) A^{1 / 2}}\right) u_{0}\right. \\
+\left(e^{-(1-t) A^{1 / 2}}-e^{-(t+1) A^{1 / 2}}\right) \\
\left.\times\left(e^{i A} u_{0}-A^{-1}\left\{\left[f(-1)-e^{i A} f(0)\right]-\int_{0}^{-1} e^{i(1+s) A} f^{\prime}(s) d s+\varphi\right\}\right)\right]
\end{gathered}
$$




$$
\begin{gathered}
+\left(I-e^{-2 A^{1 / 2}}\right)^{-1}\left(e^{-(1-t) A^{1 / 2}}-e^{-(t+1) A^{1 / 2}}\right) \\
\times \frac{1}{2} A^{-1}\left\{\left(I-e^{-2 A^{1 / 2}}\right) g(1)-\int_{0}^{1}\left(e^{-(1-s) A^{1 / 2}}-e^{-(s+1) A^{1 / 2}}\right) g^{\prime}(s) d s\right\} \\
-\frac{1}{2} A^{-1}\left\{\left[e^{-(1+t) A^{1 / 2}}-e^{-|1-t| A^{1 / 2}}\right] g(1)\right. \\
\left.-\int_{0}^{1}\left(e^{-(t+s) A^{1 / 2}}-e^{-|t-s| A^{1 / 2}}\right) g^{\prime}(s) d s\right\} .
\end{gathered}
$$

Lastly, using 2.10 and integration by parts, we get

$$
\begin{gathered}
u(0)=A^{-1 / 2} T\left(I-e^{-2 A^{1 / 2}}\right) \\
\times\left\{-i A^{-1}\left[\left(e^{-A^{1 / 2}} g(1)-g(0)\right)-\int_{0}^{1} e^{-s A^{1 / 2}} g^{\prime}(s) d s\right]-A^{-1 / 2} f(0)\right\} \\
+A^{-1 / 2} T e^{-A^{1 / 2}}\left\{2 A^{-1}\left[\left(e^{-2 i A} f(-1)-e^{-i A} f(0)\right)-\int_{0}^{-1} e^{i A(-1+s)} f^{\prime}(s) d s\right]-2 i \varphi\right. \\
\left.+\frac{i A^{-1}}{2}\left[\left(I-e^{-2 A^{1 / 2}}\right) g(1)-\int_{0}^{1}\left(e^{-(1-s) A^{1 / 2}}-e^{-(s+1) A^{1 / 2}}\right) g^{\prime}(s) d s\right]\right\} .
\end{gathered}
$$

Here, we can write

$$
g(1)=g(0)+\int_{0}^{1} g^{\prime}(s) d s \quad \text { and } \quad f(-1)=f(0)+\int_{-1}^{0} f^{\prime}(s) d s .
$$

Now, applying the operator $A$ to formulas 2.18), 2.19), 2.20 and taking their norm, we obtain

$$
\begin{gathered}
\|A u(0)\|_{H} \leq M\left[\|A \varphi\|_{H}+\|g(0)\|_{H}+\|f(0)\|_{H}+\max _{0 \leq t \leq 1}\left\|g^{\prime}(t)\right\|_{H}\right. \\
\left.+\max _{-1 \leq t \leq 0}\left\|f^{\prime}(t)\right\|_{H}\right] \\
\|A u(t)\|_{H} \leq M\left[\left\|A u_{0}\right\|_{H}+\|f(0)\|_{H}+\max _{-1 \leq t \leq 0}\left\|f^{\prime}(t)\right\|_{H}\right],-1 \leq t \leq 0, \\
\|A u(t)\|_{H} \leq M\left[\left\|A u_{0}\right\|_{H}+\|\varphi\|_{H}+\|g(0)\|_{H}+\|f(0)\|_{H}\right. \\
\left.\quad+\max _{0 \leq t \leq 1}\left\|g^{\prime}(t)\right\|_{H}+\max _{-1 \leq t \leq 0}\left\|f^{\prime}(t)\right\|_{H}\right], 0 \leq t \leq 1 .
\end{gathered}
$$

Combining estimates (2.21), 2.22) and 2.23), we obtain inequality (2.3). This completes the proof of the main theorem.

\section{Conclusion}

In conclusion, the stability estimates for the solution of problem 1.1 for the elliptic-Schrödinger equation are established. Note that some results of this paper, without proof, were presented in [17, 18.

Acknowledgments. The author would like to sincerely thank Prof. Dr. Hüseyin ÇAKALLI and Prof. Dr. Allaberen ASHYRALYEV for his valuable supports. 


\section{REFERENCES}

[1] M. S. Salahitdinov, Equations of Mixed-Composite Type, FAN, Tashkent, Uzbekistan, 1974.

[2] T. D. Drjuev, Boundary Value Problems for Equations of Mixed and Mixed-Composite Types, FAN, Tashkent, Uzbekistan, 1979.

[3] M. G. Karatopraklieva, A nonlocal boundary value problem for an equation of mixed type, Diff. Urav., 27 (1991), 68-79, in Russian.

[4] D. Bazarov and H. Soltanov, Some Local and Nonlocal Boundary Value Problems for Equations of Mixed and Mixed-Composite Types, Ylym, Ashgabat, Turkmenistan, 1995.

[5] S. N. Glazatov, Sobolev Inst. of Math. SB RAS, Preprint no: 46, 26p (1998).

[6] A. Ashyralyev, and Y. Ozdemir, On nonlocal boundary value problems hyperbolic-parabolic equations Taiwan. J. Math., 4 (2007), 1075-1089.

[7] A. Ashyralyev, and O. Gercek, Nonlocal boundary value problems of elliptic-parabolic differential and difference equations, Discrete. Dyn. Nat. Soc., 2008 (2008), 1-16.

[8] A. Ashyralyev, and A. Sirma, Nonlocal boundary value problems for Shrödinger equations, Comput. Math. Appl., 55 (2008), 392-407.

[9] A. Ashyralyev, and B. Hicdurmaz, A note on the fractional Schrödinger differential equatios, Kybernetes, 40 (2011), 736-750.

[10] A. Ashyralyev and O. Yıldırım, On multipoint nonlocal boundary value problems for hyperbolic differential and difference equations, Taiwan. J. Math., 14 (2010), 165-194.

[11] Y. Ozdemir, and M. Kucukunal, A note on nonlocal boundary value problems for hyperbolic Schrödinger equations, Abstr. Appl. Anal., 2012 (2012), 1-12.

[12] A. Ashyralyev, and O. Yildırım, A note on the second order accuracy stable difference schemes for the nonlocal boundary value hyperbolic problem, Abstr. Appl. Anal., 2012 (2012), 1-29.

[13] P. Quittner and P. Souplet, Optimal Lioville-type theorems for noncooperative elliptic Schrödinger systems and equations, Comm. Math. Phys., 311 (2012), 1-19.

[14] B. Liu and L. Ma, Symmetry results for eliiptic Schrödinger systems on half spaces, J. Math. Anal. Appl., 401 (2013), 259-268.

[15] N. Godet and N. Tzvetkov, Strichartz estimates for the periodic non-elliptic Schrödinger equation, 350 (2012), 995-958.

[16] P. Souplet, Lioville-type theorems for ellitic Schrödinger systems associated with copositive matrices, Networks \& Heterogeneous Media, 7 (2012), 697-988.

[17] Y. Ozdemir and M. Eser, Numerical solution of the elliptic-Schrödinger equation with the Dirichlet and Neumann condition, AIP Conf. Proc., 1611 (2014), 410-414.

[18] Y. Ozdemir and M. Eser, On nonlocal boundary value problems for elliptic-Schrödinger equations, AIP Conf. Proc., 1676 (2015).

YILDIRIM OZDEMIR,

Duzce University, Konuralp Campuss, Faculty of Arts and Sciences, Duzce, Turkey. Phone: $+(90) 544686$ 5722, OrCID NUMBer:0000-0003-2767-522X

Email address: yozdemir28@gmail.com

MECRA ESER,

Duzce University, Duzce, Turkey. Orcid number:0000-0002-2779-7190 